\title{
Prediction of hERG inhibition of drug discovery compounds using biomimetic HPLC measurements
}

\author{
Chrysanthos Stergiopoulos ${ }^{1 *}$, Fotios Tsopelas ${ }^{1}$ and Klara Valko ${ }^{2}$ \\ ${ }^{1}$ Laboratory of Inorganic and Analytical Chemistry, School of Chemical Engineering, National Technical University of \\ Athens \\ ${ }^{2}$ Bio-Mimetic Chromatography Ltd. Stevenage, Herts, United Kingdom
}

*Corresponding Author: Chrysanthos Stergiopoulos; E-mail: chrisxp3@hotmail.com; Tel.: +302107724022

Received: April 06, 2021; Revised: May 20, 2021; Available online: June 06, 2021

\begin{abstract}
The major causes of failure of drug discovery compounds in clinics are the lack of efficacy and toxicity. To reduce late-stage failures in the drug discovery process, it is essential to estimate early the probability of adverse effects and potential toxicity. Cardiotoxicity is one of the most often observed problems related to a compound's inhibition of the hERG channel responsible for the potassium cation flux. Biomimetic HPLC methods can be used for the early screening of a compound's lipophilicity, protein binding and phospholipid partition. Based on the published hERG pIC50 data of 90 marketed drugs and their measured biomimetic properties, a model has been developed to predict the hERG inhibition using the measured binding of compounds to alpha-1-acid-glycoprotein (AGP) and immobilised artificial membrane (IAM). A representative test set of 16 compounds was carefully selected. The training set, involving the remaining compounds, served to establish the linear model. The mechanistic model supports the hypothesis that compounds have to traverse the cell membrane and bind to the hERG ion channel to cause the inhibition. The AGP and the hERG ion channel show structural similarity, as both bind positively charged compounds with strong shape selectivity. In contrast, a good IAM partition is a prerequisite for cell membrane traversal. For reasons of comparison, a corresponding model was derived by replacing the measured biomimetic properties with calculated physicochemical properties. The model established with the measured biomimetic binding properties proved to be superior and can explain over $70 \%$ of the variance of the hERG pIC50 values.
\end{abstract}

C2021 by the authors. This article is an open-access article distributed under the terms and conditions of the Creative Commons Attribution license (http://creativecommons.org/licenses/by/4.0/).

\section{Keywords}

Cardiotoxicity; Proarrhythmia; QT Prolongation; Torsades de Pointes; hERG inhibition; IAM binding, AGP binding

\section{Introduction}

It has been recognised that the physicochemical properties of drug candidates can be related to the latestage attrition of compounds in the drug development process. The early problems with bioavailability and absorption have been successfully improved by optimising solubility and permeability [1]. Recently, toxicity and the lack of efficacy have been identified as the major cause of compound attrition in clinics. Together, preclinical toxicity and adverse events account for approximately one-third of all attrition cases [2]. 
Cardiotoxicity is one of the major causes of concern during clinical trials together with liver and central nervous system (CNS) toxicity [3]. It accounts for approximately $27 \%$ of drug development failures, and it does not seem to be restricted to specific high-risk therapeutic areas [4]. One particular focus of cardiovascular adverse effects has been drug-induced arrhythmia or "proarrhythmia" as a consequence of an increased recognition of a relationship between drug-induced QT interval prolongation and Torsades de Pointes (TdP) [5]. TdP is a dangerous type of proarrhythmia, described as a rare ventricular tachycardia with potential sudden cardiac death, which has led to approximately one-third of all drug withdrawals between 1990 and 2006 [4]. Furthermore, $15 \%$ of drugs still on the market can cause QT prolongation, and $4 \%$ are associated with TdP arrhythmia risk. Therefore, it is important to recognize a compound's cardiotoxicity potential early in the drug discovery process, not only because of the associated loss of human life or health, but also because of the enormous financial loss in investment and future revenue potential [6].

The cardiac action potential is regulated by the electrical current flows of ions across cardiomyocyte membranes. Many drugs can bind to ion channels, block ionic flow and disrupt the regulation of the action potential [7]. Upon blockade, the action potential will rest longer, which results in an increased duration of the relative QT interval that can be observed in electrocardiograph (ECG) traces. Disturbing the QT interval may lead to instability in the heart rhythm [8]. Patients with long QT syndrome (LQTS) exhibit a significant predisposition for the TdP type's cardiac arrhythmia [9]. A prolongation of the cardiac action potential and the QT interval has been associated with loss of function or drug-trapping inside the central cavity of the Kv11.1 [10] potassium channel, which is encoded by hERG (human Ether-a-go-go Related Gene) and carries the rapid delayed rectifier potassium current $\left(I_{\mathrm{Kr}}\right)[7,11]$. This channel has a tetrameric structure formed by co-assembly of four identical subunits, each composed of six helical transmembrane domains (denoted S1S6). The S4 domain contains six positive charges, typical for voltage-gated $\mathrm{K}^{+}$channels [12]. The channel pore is asymmetrical, and its dimensions change depending on its state (open-closed-inactivated). The hERG channel has been shown to interact with a wide range of drugs owing to the unique shape of the ligand-binding site, its hydrophobic character and the large vestibule of the channel $[13,14]$. The risk tolerance for QT prolongation may vary significantly depending on the dose and indication of the drug. Documented hERG-blocking activity reduces the value of a molecule, as it increases the risk of clinical failure. It has also been estimated that about $60 \%$ of drugs in development exhibit hERG block [11].

Various attempts have been made to predict the hERG inhibition potential of drugs in silico to avoid the synthesis of risky molecules [15]. When studying therapeutic areas and the safety margins regarding the free therapeutic plasma concentration of drugs [16], it was found that a wide variety of drugs, including antiarrhythmic, antibacterial, antipsychotic and pain-killer drugs showed potential risk. As toxicity, just like potency, is dose-dependent, it is essential to relate the hERG inhibitory concentration to the drugs' free therapeutic plasma concentration. It was found that a less than 30 -fold difference between the therapeutic and inhibitory concentration indicates a high risk. Redfern et al. [16] also investigated the relative value of preclinical cardiac electrophysiology data (in vitro and in vivo) for predicting the risk of TdP in drug clinical use. In vivo, telemetry experiments in non-rodents (typically dogs) are the ultimate preclinical test for cardiotoxicity. However, its high cost severely limits its use at the earlier discovery stage [17]. In vitro voltage-clamp techniques are widely used to provide real-time mechanistic information on ion channels [18]. The experiments are performed in mammalian cells transfected with the gene for $h E R G$. The overwhelming majority of predictive hERG models have been built using mammalian patch clamp data. Techniques such as fluorescence-based assays with cells transfected with hERG and radioligand (typically dofetilide or MK-499) displacement assays [17] have also been successfully used. Since the success of any 
model building depends on the quality of the biological data, it was important to carefully select reliable and informative cardiotoxicity data for a wide variety of drugs in order to develop a continuous model. As the determination of the half-maximal inhibitory concentration $\left(I_{50}\right)$ value requires measurements of inhibitory activities at multiple concentrations, the $\mathrm{IC}_{50}$ information was considered more reliable, and was selected over the inhibition type entries for positive/negative classification. Therefore, $\mathrm{IC}_{50}$ of drugs and their log unit values $\left(\mathrm{pIC}_{50}\right)$ in response to $\mathrm{hERG}$ were collected from the literature.

Certain physicochemical properties of molecules have been recognized as early indicators of potential problems with early drug discovery compounds [19]. Besides lipophilicity [20], solubility [21] and permeability, biomimetic properties such as protein [22] and phospholipid binding [23] can be measured at the early stages of the drug discovery process [24]. The chromatographic technique provides an automated, high throughput and reliable measurement of important properties of the drug discovery compounds [25] that can be used to estimate later stage in vivo properties of compounds such as the volume of distribution, the unbound volume of distribution [26] and the drug efficiency [27]. Measurements can also estimate cell membrane partition and skin penetration of compounds based on chromatographic principles $[28,29]$. Various toxicity indicators have already been related to a compound's physicochemical properties, including hERG inhibition and hepatotoxicity [30]. The toxicity potential of compounds has been studied using the immobilised artificial membrane (IAM) chromatography [31]. In this work, several chromatography-based techniques were investigated to search for the properties of the compounds that could be used to predict their toxicity, with special emphasis on cardiotoxicity.

In this study, hERG $\mathrm{pIC}_{50}$ data from a set of 90 diverse marketed drugs from a wide range of therapeutic areas and with different physicochemical properties were correlated with their measured biomimetic properties. The measurement of the biomimetic properties of the available drugs was conducted in our laboratories. Generic gradient HPLC methods were used to determine the chromatographic hydrophobicity Index ( $\mathrm{CHI})[32,33]$ using mobile phases at three different $\mathrm{pH}$ values. The protein binding of the compounds was measured using immobilised human serum albumin (HSA) [22], and alpha-1-acid-glycoprotein (AGP) stationary phases [34]. The phospholipid-binding was measured using the immobilised artificial membrane (IAM) stationary phase [23]. The aim was to establish relationships between the cardiotoxicity potential and the biomimetic binding properties of the drugs and to evaluate their predictive performance.

\section{Experimental}

The drugs were obtained from Sigma-Aldrich (Merck) and dissolved in dimethylsulfoxide (DMSO) at 10 $\mathrm{mM}$ concentration. The $10 \mu \mathrm{L}$ stock solutions were diluted down to $100 \mu \mathrm{L}$ before injecting them onto an Agilent 1100 HPLC system.

\section{CHI lipophilicity measurements}

The Chromatographic Hydrophobicity Index ( $\mathrm{CHI}$ ) was measured using the compounds' calibrated gradient retention times obtained from an Agilent 1100 HPLC fitted with a Gemini NX-C-18 column (Phenomenex Ltd Macclesfield, UK) with dimensions of $50 \times 3 \mathrm{~mm}$ and $5 \mu \mathrm{m}$ particle size. The mobile phase A was either $0.01 \mathrm{M}$ formic acid (pH 2.6), a $50 \mathrm{mM}$ ammonium acetate buffer with an adjusted $\mathrm{pH}$ of 7.4 or a $50 \mathrm{mM}$ ammonium acetate buffer with an adjusted $\mathrm{pH}$ of 10.5 . The mobile phase B was $100 \%$ acetonitrile. The flow rate was $1.0 \mathrm{~mL} / \mathrm{min}$, with starting mobile phases of $0.01 \mathrm{M}$ formic acid $(\mathrm{pH} 2.6), 50$ $\mathrm{mM}$ ammonium acetate adjusted to $\mathrm{pH} 7.4$, and $50 \mathrm{mM}$ ammonium acetate adjusted to $\mathrm{pH} 10.5$ to determine the lipophilicity of the compounds at acidic, neutral and alkaline pHs, respectively. An 
acetonitrile linear gradient was used from 0 to $100 \%$. The acetonitrile concentration reached $100 \%$ in 3.5 $\min$. The $100 \%$ acetonitrile mobile phase was maintained for an additional $1 \mathrm{~min}$ before it was returned to $0 \%$ at $4.7 \mathrm{~min}$. The gradient run cycle time was $6 \mathrm{~min}$, with an additional equilibration time of 1 min before the next injection. The standard deviation in the retention time measurements is $\pm 0.005 \mathrm{~min}$ from repeated injections. The retention time values for a standard set of compounds listed in Table1 were used to convert the drug retention times to $\mathrm{CHI}$ values.

Table 1. The $\mathrm{CHI}$ values of the calibration set of compounds at three $\mathrm{pHs}$ [33]. These values were obtained by fitting the isocratically determined $\mathrm{CHI}$ values and the gradient retention time values. The standard error ranged from 0.1 to $0.8 \mathrm{CHI}$ values. $\mathrm{CHI}$ approximates to the acetonitrile concentration when the compound elutes and can be converted to the octanol/water $\log D$ scale using $\mathrm{CHI} \log D=0.0525 * \mathrm{CHI}-1.467$ [35].

\begin{tabular}{|l|c|c|c|}
\hline Compound & $\mathbf{C H I}$ at $\mathbf{~ p H ~ 2 . 6}$ & $\mathbf{C H I}$ at $\mathbf{~ H ~} \mathbf{7 . 4}$ & $\mathbf{C H I}$ at $\mathbf{~ H ~} \mathbf{1 0 . 5}$ \\
\hline Theophylline & 17.9 & 18.4 & 5.0 \\
\hline Phenyl tetrazole & 42.2 & 23.6 & 16.0 \\
\hline Benzimidazole & 6.3 & 34.3 & 30.6 \\
\hline Colchicine & 43.9 & 45.0 & 43.9 \\
\hline Phenyl theophylline & 51.7 & 51.2 & 51.3 \\
\hline Acetophenone & 64.1 & 65.1 & 64.1 \\
\hline Indole & 72.1 & 71.5 & 72.1 \\
\hline Propiophenone & 77.4 & 77.4 & 77.4 \\
\hline Butyrophenone & 87.3 & 87.5 & 87.3 \\
\hline Valerophenone & 96.4 & 96.2 & 96.4 \\
\hline
\end{tabular}

\section{Measurements of plasma protein binding using Chiralpak HSA and AGP columns}

The protein binding measurements were carried out on Chiralpak HSA and Chiralpak AGP columns with dimensions of $3 \times 50 \mathrm{~mm}$ and $5 \mu \mathrm{m}$ particle size (Chiral Technologies Europe, France). The mobile phase was $50 \mathrm{mM}$ ammonium acetate adjusted to $\mathrm{pH} 7.4$, with a $1.2 \mathrm{~mL} / \mathrm{min}$ flow rate. The standard isopropanol (IPA) gradient reached $35 \%$ in $3.5 \mathrm{~min}$, which was maintained for $1 \mathrm{~min}$, before returning to $0 \%$ at $4.7 \mathrm{~min}$. The cycle time was $6 \mathrm{~min}$ with an additional $1 \mathrm{~min}$ re-equilibration time. The racemic warfarin showed separation of its enantiomers at retention times of 3.58 and $3.77 \mathrm{~min}$. The precision of the retention time measurements was within $\pm 0.01 \mathrm{~min}$. The calibration set of compounds and their literature $\%$ binding data which were also converted to log $k$ data are shown in Table 2.

Table 2. The protein binding data of the marketed drug molecules that were used to calibrate the retention times obtained on the chiral protein columns (Chiralpak HSA and Chiralpak AGP). The \% binding data obtained by equilibrium dialysis were converted to log $k$ data using $\log k=\log (\%$ binding/(101-\%binding)).

\begin{tabular}{lcccc}
\hline Compound name & \%HSA & log $\boldsymbol{k}$ HSA & \% AGP & log $\boldsymbol{k}$ AGP \\
\hline Warfarin & 97.9 & 1.5 & 83.2 & 0.7 \\
Paracetamol & 14.0 & -0.8 & 3.2 & -1.5 \\
Nizatidine & 20.4 & -0.6 & 37.1 & -0.2 \\
Trimethoprim & 37.6 & -0.2 & 46.2 & -0.1 \\
Propranolol & 66.6 & 0.3 & 86.0 & 0.8 \\
Carbamazepine & 75.0 & 0.5 & 65.0 & 0.3 \\
Nicardipine & 95.0 & 1.2 & 87.0 & 0.8 \\
Indomethacin & 99.5 & 1.8 & 56.0 & 0.1 \\
Diclofenac & 99.8 & 1.9 & 60.0 & 0.2 \\
\hline
\end{tabular}




\section{Measurements of phospholipid-binding at pH 7.4 using an IAM column}

The phospholipid-binding was measured using an IAM PC.DD2 column with dimensions of $100 \times 4.6 \mathrm{~mm}$ (Regis Technologies Inc., Morton Grove, IL, USA). The gradient retention times were measured using a 50 $\mathrm{mM}$ ammonium acetate mobile phase with the $\mathrm{pH}$ adjusted to 7.4. The mobile phase flow rate was 1.5 $\mathrm{mL} / \mathrm{min}$. The acetonitrile gradient was applied to reach $90 \%$ in $4.75 \mathrm{~min}$. The $90 \%$ acetonitrile concentration was maintained for an additional $0.5 \mathrm{~min}$ (to $5.25 \mathrm{~min}$ ) and returned to $0 \%$ by $5.5 \mathrm{~min}$. The cycle time was $6 \mathrm{~min}$, plus an additional 1 min equilibration time was applied while the injector prepared for the next injection. The gradient retention times were calibrated with the acetophenone homologues for which the CHI IAM values have been established using isocratic measurements [34]. Table 3 shows the calibration set of compounds and their predetermined CHI IAM values. The $\mathrm{CHI}$ Index on the IAM column (CHI IAM) approximates the acetonitrile concentration in the mobile phase when the compound elutes. $\mathrm{CHI}$ IAM values above 45 indicate strong phospholipid binding. The CHI IAM values have been converted to $\log k$ IAM values derived from the CHI IAM values using equation 1. It represents the equivalent value derived from several isocratic measurements with extrapolated log retention factors to $100 \%$ aqueous mobile phase [23]. The log $k$ IAM values can be converted to $\log K$ (IAM) values and show linear relationships with the octanol/water partition coefficients [26]. Equation 2 shows the conversion:

$$
\begin{aligned}
& \log k \text { IAM }=0.045^{*} \mathrm{CHI} \text { IAM }+0.42 \\
& \log K \text { IAM }=0.29 \mathrm{e}^{(0.045 C \text { CH IAM +0.42) }}+0.7
\end{aligned}
$$

Repeating the retention time measurements provided a standard deviation of $\pm 0.005 \mathrm{~min}$.

Table 3. The calibration set of compounds used on the IAM.PC.DD2 HPLC column and their predetermined $\mathrm{CHI}$ IAM values.

\begin{tabular}{lc}
\hline Compound & CHI IAM \\
\hline Octanophenone & 49.4 \\
Heptanophenone & 45.7 \\
Hexanophenone & 41.8 \\
Valerophenone & 37.3 \\
Butyrophenone & 32.0 \\
Propiophenone & 25.9 \\
Acetophenone & 17.2 \\
Acetanilide & 11.5 \\
Paracetamol & 2.9 \\
\hline
\end{tabular}

\section{Database search for $p / C_{50}$ values}

Assessing the risk of a blockade of the human ether à-go-go related gene potassium channels could greatly facilitate the development of therapeutic compounds and the withdrawal of hazardous marketed drugs. The development of highthroughput automated patch clamp assays has increased the amount of hERG-associated data available in public databases [17]. Integrated databases are now available using the ChEMBL and PubChem public databases. A large integrated database created by Sato et al. [36] has been used in this study. This database curates hERG-related data from in vitro assays, such as binding assays (radioligand replacement assay) and electrostatic assays (automated patch-clamp assays), in ChEMBL, PubChem, GOSTAR, NIH Chemical Genomics Center (NCGC) and hERGCentral and integrates them into the largest database about hERG inhibition. $\mathrm{IC}_{50}$ values of the compounds and their $\mathrm{plC}_{50}$ values expressed in molar concentrations were carefully searched and collected from this database, which is freely available at https://drugdesign.riken.jp/hERGdb/. Data entries using inequality signs, NULL values and value ranges were excluded. In cases of differences in the reported data for the same compound, mean values were calculated and considered for the model building while outlier values were omitted when the deviation in the results was significant (data points not falling within three standard deviations of the mean). 


\section{Calculated physicochemical properties}

ADME Boxes v.3.0 software (Pharma Algorithm) was used to calculate various physicochemical parameters of the investigated compounds such as octanol-water partition $(\log P)$ and distribution $(\log D)$ coefficients at the $\mathrm{pH}$ values of 7.4, hydrogen bond donor (HBD) and acceptor (HBA) groups, Abraham's hydrogen bond acidity (A) and basicity (B), total polar surface area (TPSA), molecular weight (MW), as well as the molecular fractions of positively charged $(\mathrm{F}+)$, negatively charged $(\mathrm{F}-)$ and zwitterionic species $(\mathrm{Fz})$ at $\mathrm{pH}=7.4$.

\section{Statistical and visualisation software}

JMP V13.0 (SAS Institute Inc) and SPSS 23.0 (IBM SPSS Statistics) were used for the statistical calculations and the stepwise regression analysis. For visualisation, Stardrop (Optibrium Ltd) chemically aware visualisation tools were used to create the plots.

\section{Results and Discussion}

Table 4 contains the collected and quality checked $\mathrm{plC}_{50}$ data of the investigated 90 drug molecules with their generic names and the measured biomimetic HPLC data. The drugs used in the training set and test set are listed separately in alphabetical order.

Table 4. The investigated marketed drugs, their hERG $\mathrm{plC}_{50}$ values and the measured biomimetic lipophilicity $\mathrm{CHI}$ $\log D$ at pH 7.4, CHI log $P$, protein binding (log $k$ HSA and $\log k$ AGP) and phospholipid partition (log $k$ IAM). The test set of compounds are listed in the second part of the table in bold.

\begin{tabular}{|c|c|c|c|c|c|c|c|c|}
\hline Drug & $\mathrm{plC}_{50}$ & $\begin{array}{c}\mathrm{CHI} \\
\log D_{7.4}\end{array}$ & $\begin{array}{c}\mathrm{CHI} \\
\log P\end{array}$ & $\begin{array}{c}\log k \\
\text { HSA }\end{array}$ & $\begin{array}{c}\log k \\
\text { AGP }\end{array}$ & $\begin{array}{l}\text { CHI } \\
\text { IAM }\end{array}$ & $\begin{array}{l}\log k \\
\text { IAM }\end{array}$ & Charge \\
\hline Amitriptyline & 5.10 & 2.65 & 5.21 & 0.91 & 1.05 & 55.27 & 2.96 & Basic \\
\hline Apomorphine & 5.59 & 1.17 & 2.66 & 1.04 & 0.83 & 39.67 & 2.24 & Weak Base \\
\hline Astemizole & 6.69 & 2.68 & 3.52 & 1.17 & 1.12 & 51.07 & 2.77 & Basic \\
\hline Atenolol & 3.00 & -0.40 & 0.64 & -1.08 & -1.83 & 15.75 & 1.14 & Basic \\
\hline Bepridil & 6.42 & 4.40 & 6.62 & 1.27 & 1.14 & 57.73 & 3.08 & Basic \\
\hline Brompheniramine & 5.61 & 1.74 & 4.27 & 0.68 & 0.68 & 51.69 & 2.8 & Basic \\
\hline Bupivacaine & 5.51 & 3.06 & 3.97 & -0.10 & 1.35 & 41.66 & 2.34 & Weak Base \\
\hline Carbamazepine & 3.98 & 1.22 & 1.33 & 0.07 & -0.85 & 23.98 & 1.53 & Neutral \\
\hline Cetirizine & 4.65 & 1.63 & 2.04 & 0.85 & 0.23 & 40.56 & 2.29 & Zwitterionic \\
\hline Chloroquine & 5.03 & 1.18 & 2.68 & 0.70 & 1.12 & 43.99 & 2.40 & Basic \\
\hline Chlorpromazine & 5.65 & -0.58 & 0.97 & 1.35 & 1.79 & 48.16 & 2.64 & Basic \\
\hline Ciprofloxacin & 3.02 & -0.09 & 0.05 & -0.54 & -1.19 & 25.39 & 1.59 & Zwitterionic \\
\hline Cisapride & 6.88 & 2.65 & 3.17 & 1.11 & 0.84 & 41.85 & 2.35 & Basic \\
\hline Citalopram & 5.27 & 1.59 & 4.32 & 0.40 & 0.44 & 48.80 & 2.66 & Basic \\
\hline Clarithromycin & 4.32 & 1.9 & 4.55 & -0.24 & 0.20 & 49.68 & 2.70 & Basic \\
\hline Clemastine & 6.69 & 3.18 & 3.18 & 1.25 & 1.18 & 60.12 & 3.19 & Basic \\
\hline Clozapine & 6.5 & 2.6 & 3.05 & 1.04 & 0.99 & 52.42 & 2.83 & Weak Base \\
\hline Desipramine & 5.36 & 1.84 & 3.49 & 0.76 & 0.94 & 53.10 & 2.86 & Basic \\
\hline Diltiazem & 4.81 & 2.76 & 3.12 & 0.36 & 0.41 & 41.95 & 2.35 & Basic \\
\hline Dolasetron & 4.65 & 1.46 & 1.46 & 0.60 & 0.69 & 33.20 & 1.95 & Weak Base \\
\hline Domperidone & 6.79 & 1.37 & 2.13 & 1.02 & 0.92 & 43.47 & 2.42 & Weak Base \\
\hline Doxazosin & 6.03 & 2.09 & 2.24 & 1.04 & 0.72 & 37.80 & 2.16 & Weak Base \\
\hline Droperidol & 6.89 & 2.26 & 2.68 & 0.98 & 1.69 & 39.31 & 2.23 & Weak Base \\
\hline
\end{tabular}


Table 4. Cont'd

\begin{tabular}{|c|c|c|c|c|c|c|c|c|}
\hline Ebastine & 6.27 & 4.96 & 6.54 & 1.62 & 1.34 & 58.65 & 3.12 & Basic \\
\hline Erythromycin & 4.43 & 1.31 & 2.60 & -0.22 & 0.32 & 38.79 & 2.20 & Basic \\
\hline Fexofenadine & 4.61 & 1.28 & 1.91 & 0.45 & 0.02 & 32.24 & 1.90 & Zwitterionic \\
\hline Fluvoxamine & 5.2 & 2.01 & 3.34 & 0.4 & 0.59 & 50.22 & 2.73 & Basic \\
\hline Glibenclamide & 4.29 & 2.07 & 3.17 & 1.52 & 0.39 & 32.63 & 1.93 & Acidic \\
\hline Glimepiride & 4.13 & 2.42 & 3.25 & 1.50 & 0.40 & 30.76 & 1.83 & Basic \\
\hline Granisetron & 4.65 & 0.79 & 2.50 & 0.34 & 0.52 & 45.95 & 2.53 & Basic \\
\hline Imipramine & 5.40 & 2.05 & 4.60 & 0.95 & 0.96 & 39.44 & 2.23 & Basic \\
\hline Isradipine & 5.71 & 3.25 & 3.29 & 1.19 & 1.32 & 38.76 & 2.21 & Neutral \\
\hline Ketoconazole & 5.49 & 2.66 & 2.84 & 1.18 & 0.75 & 37.86 & 2.16 & Weak Base \\
\hline Levobupivacaine & 5.48 & 3.25 & 4.20 & 0.13 & 1.25 & 41.83 & 2.34 & Weak Base \\
\hline Levofloxacin & 2.93 & 0.54 & 0.54 & -0.34 & -0.74 & 28.55 & 1.73 & Zwitterionic \\
\hline Lidocaine & 3.58 & 2.65 & 3.01 & -0.71 & 0.17 & 32.38 & 1.91 & Weak Base \\
\hline Lomefloxacin & 3.93 & -0.14 & -0.14 & -0.10 & -1.01 & 37.89 & 2.16 & Zwitterionic \\
\hline Loratadine & 4.90 & 3.86 & 4.25 & 1.38 & 1.34 & 44.30 & 2.46 & Weak Base \\
\hline Lovastatin & 5.16 & 4.09 & 4.24 & 1.24 & 0.87 & 44.38 & 2.46 & Neutral \\
\hline Maprotiline & 5.17 & 2.03 & 4.79 & 0.77 & 0.99 & 56.73 & 3.03 & Basic \\
\hline Mefloquine & 5.45 & 2.18 & 4.22 & 1.38 & 1.11 & 41.65 & 2.29 & Basic \\
\hline Metoclopramide & 5.27 & 0.5 & 1.91 & 0.15 & 0.23 & 40.38 & 2.28 & Basic \\
\hline Mibefradil & 5.88 & 2.96 & 3.99 & 1.10 & 1.11 & 55.18 & 2.96 & Basic \\
\hline Miconazole & 5.68 & 4.64 & 4.70 & 1.54 & 1.26 & 53.72 & 2.89 & Weak Base \\
\hline Moxifloxacin & 3.83 & 0.69 & 0.81 & 0.54 & -0.46 & 31.61 & 1.84 & Zwitterionic \\
\hline Nicotine & 3.61 & 0.12 & 1.20 & -0.03 & -0.98 & 13.79 & 1.05 & Weak Base \\
\hline Nifedipine & 3.96 & 2.59 & 2.71 & 0.67 & 0.27 & 25.12 & 1.58 & Basic \\
\hline Nitrendipine & 5.10 & 3.15 & 3.19 & 1.17 & 0.69 & 39.36 & 2.23 & Neutral \\
\hline Ofloxacin & 2.93 & 0.53 & 0.53 & -0.40 & -0.81 & 26.17 & 1.62 & Zwitterionic \\
\hline Olanzapine & 5.06 & 1.96 & 2.76 & 0.76 & 0.72 & 49.74 & 2.71 & Weak Base \\
\hline Ondansetron & 5.73 & 1.33 & 1.66 & 0.54 & 1.30 & 39.01 & 2.22 & Weak Base \\
\hline Pergolide & 6.52 & 2.47 & 3.81 & 0.73 & 1.04 & 52.73 & 2.85 & Basic \\
\hline Perphenazine & 5.88 & 2.92 & 3.50 & 1.39 & 1.13 & 47.64 & 2.61 & Weak Base \\
\hline Phenytoin & 3.71 & 1.73 & 1.85 & 0.69 & 0.15 & 29.66 & 1.78 & Weak Acid \\
\hline Pimozide & 6.43 & 3.03 & 3.89 & 1.41 & 1.47 & 52.31 & 2.83 & Weak Base \\
\hline Prazosin & 5.22 & 1.09 & 1.29 & 0.81 & 0.53 & 26.32 & 1.63 & Weak Base \\
\hline Procainamide & 3.86 & -0.59 & 0.95 & -0.71 & -0.54 & 19.30 & 1.29 & Basic \\
\hline Propafenone & 5.96 & 2.07 & 3.47 & 0.84 & 0.92 & 46.04 & 2.54 & Basic \\
\hline Propiverine & 5.22 & 3.84 & 5.05 & 1.01 & 0.94 & 58.60 & 3.12 & Basic \\
\hline Propranolol & 5.03 & 1.63 & 3.03 & 0.47 & 0.84 & 42.08 & 2.36 & Basic \\
\hline Pyrilamine & 5.18 & 1.72 & 3.32 & 0.44 & 0.49 & 46.62 & 2.56 & Basic \\
\hline Quetiapine & 5.21 & 2.55 & 2.69 & 0.92 & 0.97 & 39.38 & 2.23 & Weak Base \\
\hline Quinidine & 5.51 & 1.25 & 2.38 & 0.54 & 0.68 & 49.78 & 2.71 & Basic \\
\hline Risperidone & 6.00 & 1.46 & 2.18 & 0.55 & 0.63 & 36.31 & 2.09 & Weak Base \\
\hline Ritonavir & 5.09 & 3.25 & 3.41 & 1.22 & 0.64 & 38.64 & 2.2 & Neutral \\
\hline Roxithromycin & 4.44 & 1.95 & 3.70 & -0.07 & 0.60 & 51.33 & 2.78 & Basic \\
\hline Saquinavir & 5.82 & 3.31 & 3.39 & 1.22 & 1.59 & 42.17 & 2.36 & Weak Base \\
\hline Sotalol & 3.57 & -0.39 & -0.35 & -0.75 & -1.41 & 21.90 & 1.43 & Basic \\
\hline
\end{tabular}


Table 4. Cont'd

\begin{tabular}{|c|c|c|c|c|c|c|c|c|}
\hline Sulfamethoxazole & 2.66 & -0.42 & 1.03 & 0.4 & -1.57 & 13.19 & 1.01 & Acidic \\
\hline Tamsulosin & 4.89 & 1.43 & 2.01 & 0.36 & 0.60 & 34.32 & 2.00 & Basic \\
\hline Thioridazine & 6.30 & 3.02 & 5.46 & 1.33 & 1.30 & 70.24 & 3.65 & Basic \\
\hline Tolterodine & 6.23 & 2.00 & 4.95 & 0.55 & 0.79 & 52.45 & 2.83 & Basic \\
\hline Trifluoperazine & 5.72 & 3.8 & 5.14 & 1.52 & 1.19 & 68.00 & 3.55 & Basic \\
\hline Verapamil & 5.95 & 2.66 & 3.82 & 1.00 & 0.58 & 44.82 & 2.48 & Basic \\
\hline Alfuzosin & 4.28 & 0.79 & 1.31 & 0.08 & 0.32 & 34.60 & 1.98 & Weak Base \\
\hline Desloratadine & 5.75 & 1.32 & 4.03 & 0.85 & 1.05 & 54.56 & 2.93 & Basic \\
\hline Diphenhydramine & 4.94 & 1.59 & 3.61 & 0.33 & 0.60 & 35.63 & 2.06 & Basic \\
\hline Dofetilide & 5.72 & 1.18 & 1.12 & -0.05 & 0.59 & 33.10 & 1.94 & Weak Base \\
\hline Flecainide & 5.25 & 1.68 & 3.01 & -0.05 & 0.16 & 41.51 & 2.33 & Basic \\
\hline Fluoxetine & 5.73 & 2.15 & 3.80 & 0.99 & 0.99 & 54.86 & 2.94 & Basic \\
\hline Indomethacin & 3.72 & 1.47 & 3.31 & 1.69 & 0.18 & 30.11 & 1.81 & Acidic \\
\hline Irbesartan & 4.71 & 1.39 & 2.00 & 1.30 & 1.10 & 26.14 & 1.62 & Zwitterionic \\
\hline Lamotrigine & 3.55 & 0.86 & 0.81 & 0.16 & -0.33 & 23.25 & 1.49 & Weak Base \\
\hline Metoprolol & 3.84 & 0.77 & 1.86 & -0.72 & -0.27 & 35.38 & 2.05 & Basic \\
\hline Protriptyline & 5.30 & 1.72 & 4.70 & 0.69 & 1.03 & 51.59 & 2.79 & Weak Base \\
\hline Sildenafil & 4.61 & 2.59 & 2.62 & 0.87 & 0.42 & 35.00 & 2.03 & Weak Base \\
\hline Spironolactone & 4.64 & 2.76 & 2.97 & 0.78 & 0.50 & 36.93 & 2.12 & Neutral \\
\hline Trazodone & 5.30 & 2.51 & 2.59 & 1.09 & 0.46 & 32.7 & 1.93 & Weak Base \\
\hline Trimethoprim & 3.62 & -1.60 & 0.42 & -0.05 & -0.07 & 12.83 & 1.01 & Basic \\
\hline Ziprasidone & 6.44 & 2.98 & 2.96 & 1.42 & 1.09 & 47.92 & 2.62 & Weak Base \\
\hline
\end{tabular}

Table 5 contains the calculated physicochemical properties of the investigated compounds. The test set listed separately in alphabetical order in the last part of the table.

Table 5. The calculated physicochemical properties of the compounds. $M W$ is the molecular weight, HBD and HBA are the numbers of $\mathrm{H}$-bond donor and acceptor groups, respectively, TPSA is the topological polar surface area, $\log P$ and $\log D$ are the logarithm of the calculated octanol/water partition coefficient of the neutral form and the combined ionised form of the molecules at $\mathrm{pH} 7.4, \mathrm{~F}+, \mathrm{F}$ - and $\mathrm{Fz}$ are the calculated fractions of the positive, negative and zwitterionic charges at physiological $\mathrm{pH}(\mathrm{pH} 7.4), \mathrm{A}$ and $\mathrm{B}$ are the Abraham $\mathrm{H}$-bond acidity and $\mathrm{B}$ bond basicity parameters.

\begin{tabular}{lccccccccccc}
\hline Drug & MW & HBD & HBA & TPSA & $\log \boldsymbol{P}$ & $\log \boldsymbol{D}$ & F+ & F- & Fz & A & B \\
\hline Amitriptyline & 277.41 & 0 & 1 & 3.2 & 5.04 & 3.70 & 0.98 & 0.00 & 0.00 & 0.00 & 1.00 \\
Apomorphine & 267.32 & 2 & 3 & 43.7 & 2.49 & 2.16 & 0.62 & 0.00 & 0.02 & 0.77 & 1.10 \\
Astemizole & 458.57 & 1 & 5 & 42.3 & 5.70 & 4.54 & 0.98 & 0.00 & 0.00 & 0.13 & 1.64 \\
Atenolol & 266.34 & 4 & 5 & 84.6 & 0.16 & -1.89 & 0.99 & 0.00 & 0.00 & 0.69 & 2.00 \\
Bepridil & 366.54 & 0 & 3 & 15.7 & 6.31 & 4.9 & 0.97 & 0.00 & 0.00 & 0.00 & 1.32 \\
Brompheniramine & 319.24 & 0 & 2 & 16.1 & 2.88 & 1.64 & 0.99 & 0.00 & 0.00 & 0.00 & 1.02 \\
Bupivacaine & 288.43 & 1 & 3 & 32.3 & 3.41 & 3.73 & 0.78 & 0.00 & 0.00 & 0.26 & 1.19 \\
Carbamazepine & 236.27 & 2 & 3 & 46.3 & 2.30 & 2.58 & 0.00 & 0.00 & 0.00 & 0.53 & 1.10 \\
Cetirizine & 388.89 & 1 & 5 & 53.0 & 1.70 & 0.34 & 0.00 & 0.22 & 0.78 & 0.57 & 1.76 \\
Chloroquine & 319.87 & 1 & 3 & 28.2 & 4.63 & 2.60 & 1.00 & 0.00 & 0.00 & 0.13 & 1.29 \\
Chlorpromazine & 318.86 & 0 & 2 & 31.8 & 5.35 & 3.34 & 0.99 & 0.00 & 0.00 & 0.00 & 0.94 \\
Ciprofloxacin & 331.34 & 2 & 6 & 72.9 & -1.08 & -3.03 & 0.05 & 0.03 & 0.91 & 0.73 & 1.85 \\
Cisapride & 465.94 & 3 & 7 & 86.1 & 4.20 & 2.49 & 0.98 & 0.00 & 0.00 & 0.50 & 2.17 \\
\hline
\end{tabular}


Table 5. Cont'd

\begin{tabular}{|c|c|c|c|c|c|c|c|c|c|c|c|}
\hline Citalopram & 324.39 & 0 & 3 & 36.3 & 3.76 & 0.99 & 0.99 & 0.00 & 0.00 & 0.00 & 1.08 \\
\hline Clarithromycin & 747.95 & 4 & 14 & 182.9 & 3.16 & 1.16 & 0.95 & 0.00 & 0.00 & 0.80 & 4.49 \\
\hline Clemastine & 343.89 & 0 & 2 & 12.5 & 5.79 & 3.63 & 0.99 & 0.00 & 0.00 & 0.00 & 0.97 \\
\hline Clozapine & 326.82 & 1 & 4 & 30.9 & 3.32 & 4.68 & 0.58 & 0.00 & 0.00 & 0.18 & 1.44 \\
\hline Desipramine & 266.38 & 1 & 2 & 15.3 & 4.90 & 1.57 & 1.00 & 0.00 & 0.00 & 0.09 & 0.91 \\
\hline Diltiazem & 414.52 & 0 & 6 & 84.4 & 2.70 & 1.97 & 1.00 & 0.00 & 0.00 & 0.00 & 2.12 \\
\hline Dolasetron & 324.37 & 1 & 5 & 62.4 & 2.70 & 2.66 & 0.10 & 0.00 & 0.00 & 0.31 & 1.52 \\
\hline Domperidone & 425.92 & 2 & 7 & 67.9 & 4.05 & 3.49 & 0.85 & 0.00 & 0.00 & 0.72 & 1.83 \\
\hline Doxazosin & 451.47 & 2 & 10 & 112.3 & 2.07 & 1.97 & 0.22 & 0.00 & 0.00 & 0.23 & 2.60 \\
\hline Droperidol & 379.43 & 1 & 5 & 52.7 & 3.50 & 2.61 & 0.85 & 0.00 & 0.00 & 0.33 & 1.67 \\
\hline Ebastine & 469.66 & 0 & 3 & 29.5 & 7.55 & 6.14 & 0.97 & 0.00 & 0.00 & 0.00 & 1.41 \\
\hline Erythromycin & 733.92 & 5 & 14 & 193.9 & 2.54 & 0.65 & 0.95 & 0.00 & 0.00 & 1.05 & 4.63 \\
\hline Fexofenadine & 501.65 & 3 & 5 & 81.0 & 4.35 & 1.83 & 0.00 & 0.04 & 0.97 & 1.20 & 2.12 \\
\hline Fluvoxamine & 318.33 & 2 & 4 & 56.8 & 3.63 & 2.32 & 0.96 & 0.00 & 0.00 & 0.23 & 1.14 \\
\hline Glibenclamide & 494.00 & 3 & 8 & 122.0 & 4.02 & 1.83 & 0.00 & 0.99 & 0.00 & 0.85 & 2.01 \\
\hline Glimepiride & 490.62 & 3 & 9 & 133.1 & 4.25 & 2.05 & 0.99 & 0.00 & 0.00 & 0.75 & 2.15 \\
\hline Granisetron & 312.41 & 1 & 5 & 50.2 & 0.79 & -1.31 & 0.99 & 0.00 & 0.00 & 0.26 & 1.56 \\
\hline Imipramine & 280.41 & 0 & 2 & 4.8 & 4.28 & 2.61 & 0.99 & 0.00 & 0.00 & 0.00 & 1.15 \\
\hline Isradipine & 371.39 & 1 & 8 & 103.6 & 4.18 & 1.48 & 0.00 & 0.00 & 0.00 & 0.13 & 1.79 \\
\hline Ketoconazole & 531.43 & 0 & 8 & 69.1 & 4.34 & 3.98 & 0.15 & 0.00 & 0.00 & 0.00 & 2.22 \\
\hline Levobupivacaine & 288.43 & 1 & 3 & 32.3 & 4.35 & 3.73 & 0.78 & 0.00 & 0.00 & 0.26 & 1.19 \\
\hline Levofloxacin & 361.37 & 1 & 7 & 73.3 & -0.24 & -2.34 & 0.05 & 0.10 & 0.85 & 0.57 & 2.05 \\
\hline Lidocaine & 234.34 & 1 & 3 & 32.3 & 2.26 & 2.44 & 0.78 & 0.00 & 0.00 & 0.12 & 1.21 \\
\hline Lomefloxacin & 351.35 & 2 & 6 & 72.9 & -0.80 & -3.43 & 0.05 & 0.03 & 0.91 & 0.73 & 1.81 \\
\hline Loratadine & 382.88 & 0 & 4 & 42.4 & 5.20 & 4.94 & 0.00 & 0.00 & 0.00 & 0.00 & 1.14 \\
\hline Lovastatin & 404.54 & 1 & 5 & 72.8 & 4.26 & 4.40 & 0.00 & 0.00 & 0.00 & 0.31 & 1.44 \\
\hline Maprotiline & 277.41 & 1 & 1 & 12.0 & 4.85 & 1.48 & 1.00 & 0.00 & 0.00 & 0.13 & 0.68 \\
\hline Mefloquine & 378.31 & 2 & 3 & 45.2 & 3.28 & 1.50 & 0.99 & 0.00 & 0.00 & 0.38 & 1.22 \\
\hline Metoclopramide & 299.80 & 3 & 5 & 67.6 & 1.40 & 0.36 & 0.99 & 0.00 & 0.00 & 0.50 & 1.63 \\
\hline Mibefradil & 495.63 & 1 & 6 & 67.5 & 4.97 & 2.37 & 1.00 & 0.00 & 0.00 & 0.35 & 1.80 \\
\hline Miconazole & 416.13 & 0 & 3 & 27.1 & 5.34 & 5.45 & 0.15 & 0.00 & 0.00 & 0.00 & 0.79 \\
\hline Moxifloxacin & 401.43 & 2 & 7 & 82.1 & -0.08 & -2.87 & 0.05 & 0.01 & 0.94 & 0.72 & 2.04 \\
\hline Nicotine & 162.23 & 0 & 2 & 16.1 & 0.75 & -0.12 & 0.88 & 0.00 & 0.00 & 0.00 & 0.91 \\
\hline Nifedipine & 346.33 & 1 & 8 & 113.4 & 3.27 & 1.12 & 1.00 & 0.00 & 0.00 & 0.23 & 1.45 \\
\hline Nitrendipine & 360.36 & 1 & 8 & 113.5 & 4.15 & 1.60 & 0.00 & 0.00 & 0.00 & 0.13 & 1.54 \\
\hline Ofloxacin & 361.37 & 1 & 7 & 73.3 & -0.39 & -2.34 & 0.05 & 0.10 & 0.85 & 0.57 & 2.05 \\
\hline Olanzapine & 312.44 & 1 & 4 & 59.1 & 3.00 & 4.05 & 0.58 & 0.00 & 0.00 & 0.13 & 1.45 \\
\hline Ondansetron & 293.36 & 0 & 4 & 39.8 & 1.96 & 1.09 & 0.88 & 0.00 & 0.00 & 0.00 & 1.53 \\
\hline Pergolide & 314.49 & 1 & 2 & 44.3 & 4.01 & 2.97 & 0.92 & 0.00 & 0.00 & 0.31 & 1.01 \\
\hline Perphenazine & 403.97 & 1 & 4 & 55.3 & 3.69 & 3.86 & 0.74 & 0.00 & 0.00 & 0.23 & 1.84 \\
\hline Phenytoin & 252.27 & 2 & 4 & 58.2 & 2.15 & 1.90 & 0.00 & 0.10 & 0.00 & 0.85 & 1.00 \\
\hline Pimozide & 461.54 & 1 & 4 & 35.6 & 6.30 & 5.49 & 0.85 & 0.00 & 0.00 & 0.33 & 1.44 \\
\hline Prazosin & 383.40 & 2 & 9 & 107.0 & 0.45 & 0.35 & 0.22 & 0.00 & 0.00 & 0.23 & 2.17 \\
\hline Procainamide & 235.32 & 2 & 4 & 58.4 & 0.88 & -0.83 & 0.99 & 0.00 & 0.00 & 0.50 & 1.45 \\
\hline Propafenone & 341.44 & 2 & 4 & 58.6 & 3.41 & 1.26 & 0.99 & 0.00 & 0.00 & 0.29 & 1.67 \\
\hline
\end{tabular}


Table 5. Cont'd

\begin{tabular}{|c|c|c|c|c|c|c|c|c|c|c|c|}
\hline Propiverine & 367.48 & 0 & 4 & 38.8 & 4.01 & 1.53 & 1.00 & 0.00 & 0.00 & 0.00 & 1.31 \\
\hline Propranolol & 259.34 & 2 & 3 & 41.5 & 3.09 & 0.89 & 0.99 & 0.00 & 0.00 & 0.17 & 1.42 \\
\hline Pyrilamine & 285.38 & 0 & 4 & 28.6 & 3.27 & 1.63 & 0.97 & 0.00 & 0.00 & 0.00 & 1.59 \\
\hline Quetiapine & 383.51 & 1 & 5 & 73.6 & 2.27 & 1.91 & 0.58 & 0.00 & 0.00 & 0.23 & 2.01 \\
\hline Quinidine & 324.42 & 1 & 4 & 45.6 & 2.64 & 1.06 & 0.95 & 0.00 & 0.00 & 0.23 & 1.81 \\
\hline Risperidone & 410.48 & 0 & 6 & 61.9 & 3.04 & 1.09 & 0.88 & 0.00 & 0.00 & 0.00 & 1.70 \\
\hline Ritonavir & 720.94 & 4 & 11 & 202.3 & 5.64 & 5.64 & 0.00 & 0.00 & 0.00 & 0.88 & 3.14 \\
\hline Roxithromycin & 837.04 & 5 & 17 & 216.9 & 3.79 & 1.00 & 0.95 & 0.00 & 0.00 & 1.05 & 5.12 \\
\hline Saquinavir & 670.84 & 6 & 11 & 166.8 & 3.77 & 3.67 & 0.22 & 0.00 & 0.00 & 1.46 & 3.89 \\
\hline Sotalol & 272.36 & 3 & 5 & 86.8 & 0.24 & -1.35 & 0.91 & 0.00 & 0.08 & 0.74 & 1.75 \\
\hline Sulfamethoxazole & 253.28 & 3 & 6 & 106.6 & 0.89 & -0.85 & 0.00 & 0.98 & 0.00 & 0.59 & 1.21 \\
\hline Tamsulosin & 408.51 & 2 & 7 & 119.3 & 2.38 & 1.07 & 0.95 & 0.00 & 0.00 & 0.59 & 2.11 \\
\hline Thioridazine & 370.58 & 0 & 2 & 57.1 & 5.90 & 4.17 & 0.99 & 0.00 & 0.00 & 0.00 & 1.13 \\
\hline Tolterodine & 339.51 & 1 & 2 & 23.5 & 5.98 & 3.49 & 1.00 & 0.00 & 0.00 & 0.50 & 1.08 \\
\hline Trifluoperazine & 407.50 & 0 & 3 & 35.0 & 5.03 & 4.37 & 0.85 & 0.00 & 0.00 & 0.00 & 1.50 \\
\hline Verapamil & 454.60 & 0 & 6 & 64.0 & 3.83 & 3.17 & 0.98 & 0.00 & 0.00 & 0.00 & 1.89 \\
\hline Alfuzosin & 389.45 & 3 & 9 & 111.8 & -0.23 & -0.85 & 0.78 & 0.00 & 0.00 & 0.84 & 2.24 \\
\hline Desloratadine & 310.83 & 1 & 2 & 24.9 & 4.13 & 2.42 & 0.98 & 0.00 & 0.00 & 0.13 & 0.99 \\
\hline Diphenhydramine & 255.35 & 0 & 2 & 12.5 & 3.40 & 1.82 & 0.97 & 0.00 & 0.00 & 0.00 & 0.95 \\
\hline Dofetilide & 441.57 & 2 & 8 & 121.6 & 2.41 & 1.06 & 0.88 & 0.00 & 0.08 & 0.72 & 2.16 \\
\hline Flecainide & 414.34 & 2 & 5 & 59.6 & 3.78 & 0.32 & 0.99 & 0.00 & 0.00 & 0.41 & 1.32 \\
\hline Fluoxetine & 309.33 & 1 & 2 & 21.3 & 4.50 & 2.44 & 1.00 & 0.00 & 0.00 & 0.13 & 0.78 \\
\hline Indomethacin & 357.79 & 1 & 5 & 68.5 & 4.27 & 0.71 & 0.00 & 1.00 & 0.00 & 0.57 & 1.57 \\
\hline Irbesartan & 428.53 & 1 & 7 & 87.1 & 4.72 & 2.22 & 0.00 & 0.00 & 1.00 & 0.56 & 1.78 \\
\hline Lamotrigine & 256.09 & 4 & 5 & 90.7 & 2.63 & 2.62 & 0.01 & 0.00 & 0.00 & 0.35 & 0.96 \\
\hline Metoprolol & 267.36 & 2 & 4 & 50.7 & 1.88 & -0.43 & 0.99 & 0.00 & 0.00 & 0.17 & 1.76 \\
\hline Protriptyline & 263.38 & 1 & 1 & 12.0 & 4.91 & 4.04 & 0.88 & 0.00 & 0.00 & 0.13 & 0.73 \\
\hline Sildenafil & 474.58 & 1 & 10 & 117.5 & 2.73 & 2.47 & 0.37 & 0.06 & 0.04 & 0.26 & 2.68 \\
\hline Spironolactone & 416.57 & 0 & 4 & 85.7 & 2.52 & 2.52 & 0.00 & 0.00 & 0.00 & 0.00 & 1.63 \\
\hline Trazodone & 371.86 & 0 & 6 & 42.4 & 3.80 & 4.60 & 0.22 & 0.00 & 0.00 & 0.00 & 1.92 \\
\hline Trimethoprim & 290.32 & 4 & 7 & 105.5 & 1.28 & 0.71 & 0.47 & 0.00 & 0.00 & 0.28 & 1.62 \\
\hline Ziprasidone & 412.94 & 1 & 5 & 46.7 & 4.6 & 4.14 & 0.64 & 0.00 & 0.00 & 0.48 & 1.65 \\
\hline
\end{tabular}

\section{Selection of the test set of compounds}

Constructed toxicity models require external validation to prove their predictive ability. Hence, a test set, usually consisting of about $20 \%$ of the entire set, is necessary to evaluate the established models in terms of their predictive performance [37]. For that reason, a principal component analysis using the calculated physicochemical properties of the compounds was performed. By plotting the first two principal components (Figure 1), four compounds from each quadrant were selected by taking into account the compounds' therapeutic areas to ensure the test set's diversity. The remaining compounds were used for modelling as the training set. Table 6 shows the therapeutic areas of the compounds selected as the test set. 


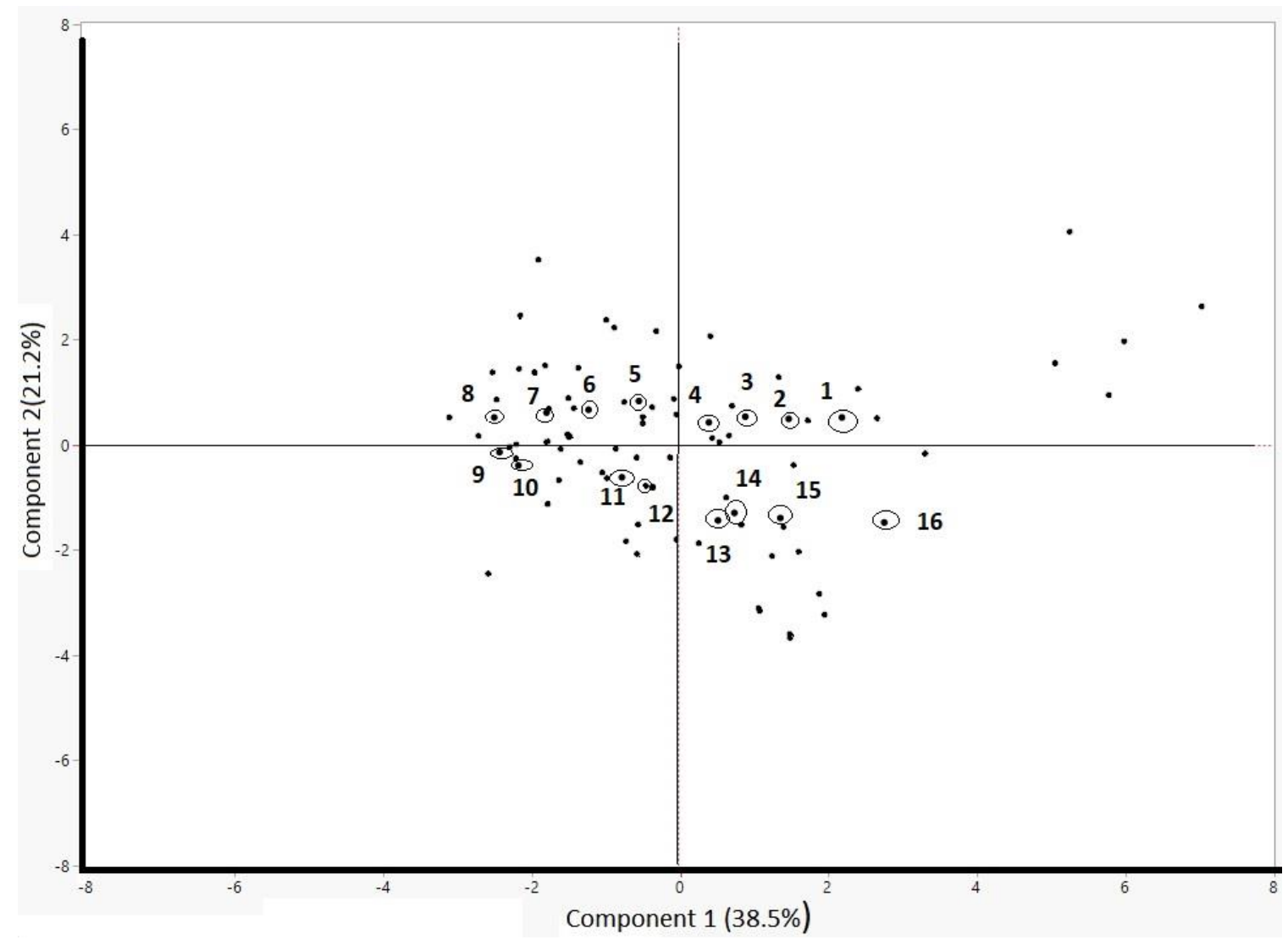

Figure 1 The score plot from the principal component analysis on the calculated properties. Compounds served as the test set are marked in red, as follows: (1) dofetilide, (2) sildenafil, (3) irbesartan, (4) flecainide, (5) ziprasidone, (6) trazodone, (7) fluoxetine, (8) protryptiline, (9) diphenhydramine, (10) desloratanide, (11) spironolactone, (12) metoprolol, (13) lamotrigine, (14) indomethacin, (15) trimethoprim, (16) alfuzosin.

Table 6. Compounds selected for the test set and their indications.

\begin{tabular}{lc}
\hline \multicolumn{1}{c}{ Pharmaceutical } & Drug class \\
\hline Alfuzosin & alpha blocker \\
Desloratadine & tricyclic antihistamine \\
Diphenhydramine & antihistamine \\
Dofetilide & class III antiarrhythmic agent \\
Flecainide & class I antiarrhythmic agent \\
Fluoxetine & selective serotonin reuptake inhibitor (SSRI) antidepressant \\
Indomethacin & non-steroidal anti-inflammatory (NSAID) \\
Irbesartan & angiotensin receptor blocker (ARB) \\
Lamotrigine & antiepileptic/anticonvulsant \\
Metoprolol & class II antiarrhythmic agent \\
Protriptyline & tricyclic antidepressant \\
Sildenafil & phosphodiesterase (PDE) inhibitor \\
Spironolactone & aldosterone antagonist \\
Trazodone & serotonin antagonist and reuptake inhibitor (SARI) antidepressant \\
Trimethoprim & antibacterial \\
Ziprasidone & atypical antipsychotic \\
\hline
\end{tabular}

Using stepwise regression analysis on the training set, a model was built using only the measured properties listed in Table 4. The best model can be described by equation 3: 


$$
\begin{aligned}
& \mathrm{pIC}_{50}=3.63( \pm 0.37)+0.88( \pm 0.12) * \log k \mathrm{AGP}+0.42( \pm 0.18) * \log k \mathrm{IAM} \\
& \left(\mathrm{R}=0.850, \mathrm{R}^{2}=0.722, \mathrm{R}^{2} \text { adj }=0.714, \mathrm{~N}=74, \mathrm{~s}=0.553, \mathrm{~F}=92.1\right)
\end{aligned}
$$

$\mathrm{R}$ is the correlation coefficient, $\mathrm{N}$ is the number of compounds, $\mathrm{s}$ is the standard error of the estimate, $\mathrm{F}$ is the Fisher test value. The IAM binding and AGP binding variables are highly significant and showed a week intercorrelation $\left(R^{2}=0.42\right)$. Therefore, the equation was recalculated using Partial Least Squares regression (PLS) and the same coefficients and intercept were obtained.

The best model using only in silico calculated properties, the molecular weight (MW), the number of $\mathrm{H}$ bond donors (HBD) and acceptor groups (HBA), the polar surface (TPSA), the logarithm of the calculated octanol/water partition coefficient of the neutral form and the combined ionised form of the molecules at $\mathrm{pH} 7.4(\log P$ and $\log D)$, calculated fractions of the positive, negative and zwitterionic charges at physiological $\mathrm{pH}(\mathrm{F}+, \mathrm{F}-, \mathrm{Fz})$, and the Abraham $\mathrm{H}$-bond acidity and $\mathrm{B}$-bond basicity parameters $(\mathrm{A}, \mathrm{B})$ can be described by equation 4 .

$$
\begin{aligned}
& \mathrm{plC}_{50}=3.94( \pm 0.17)+0.38( \pm 0.05) * \log P-1.49( \pm 0.51)^{*} \mathrm{~F}- \\
& \left(\mathrm{R}=0.740, \mathrm{R}^{2}=0.547, \mathrm{R}_{\text {ad }}^{2}=0.534, \mathrm{~N}=74, \mathrm{~s}=0.706, \mathrm{~F}=42.9\right)
\end{aligned}
$$

It was found that $\log P$ is correlated better than $\log D$ with $\mathrm{plC}_{50}$ and only negatively charged molecular fraction F- stands as statistically significant additional physicochemical parameter next to $\log P$. The statistical insignificance of $\mathrm{F}+$ may be attributed to the fact that its positive sign due to ionization counterbalanced with its positive influence to $\mathrm{plC}_{50}$. The models' statistical parameters are much worse when compared to the model using measured AGP binding (log $k$ AGP) data and membrane partition ( $\log k$ IAM) data.

The estimated $\mathrm{pIC}_{50}$ values of the test set have been calculated using equation 3 and plotted in Figure 2 . The test set compounds are marked with larger circles.

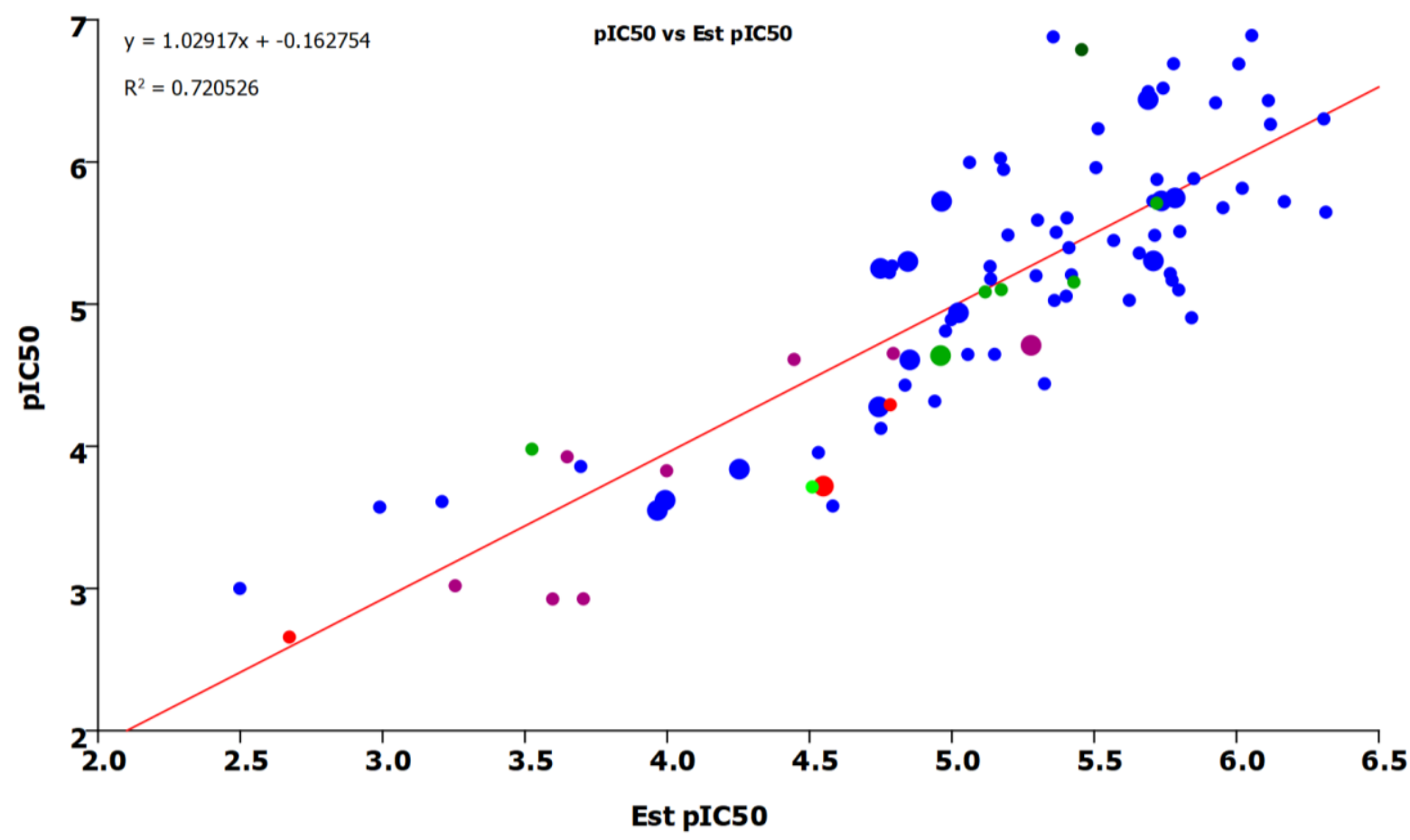

Figure 2 shows the literature $\mathrm{hERG} \mathrm{pIC}_{50}$ data and the back-calculated $\mathrm{pIC}_{50}$ data using equation 3. Blue circles mark positively charged compounds at $\mathrm{pH} 7.4$; red circles mark negatively charged compounds at $\mathrm{pH} 7.4$. The green circles mark neutral compounds at $\mathrm{pH} 7.4$; green circle's shade reflects the presence of weak acidic (lighter green) or weak basic (darker green) groups in the molecules; purple circles indicate compounds with zwitterionic character at $\mathrm{pH} 7.4$ 
It can be seen in Figure 2 that the majority of the positively charged compounds show a $\mathrm{plC}_{50}$ value greater than 5 log units in the in vitro hERG experiments. Acidic and zwitterionic compounds show only weak hERG inhibition. It is also interesting to note that the AGP binding data showed a strong correlation with the compounds binding to hERG channel receptors. The explanation for this may lie in the similarity of the two proteins. It was found [38] that the AGP binding site can be featured as a funnel-like structure. The side of the funnel represents a lipophilic region. The funnel's top width provides a steric hindrance for molecules wider than the funnel, while at the narrow end of the funnel are the negatively charged sialic acid residues that bind the positive charges if they fit into the deep end of the funnel. The structure is illustrated in Figure 3. The IAM binding, which shows the compound's membrane partition, was also significant in the model, which is not surprising as the ion channel receptor is in the membrane. The compound needs to have high membrane affinity to be able to approach the channel. The positive charge also promotes the binding to the negatively charged surface. Both the AGP and IAM stationary phases show strong shape selectivity, which is also essential to hERG inhibition. Although a wide variety of molecules show high $\mathrm{plC}_{50}$ values the shape of the molecule is very important because of the channel opening's well-defined size. This fact reduces the power of the in silico models if only 2D descriptors are used in the model building. As a result, the 3D description of the molecules would probably enhance the success of in silico models.
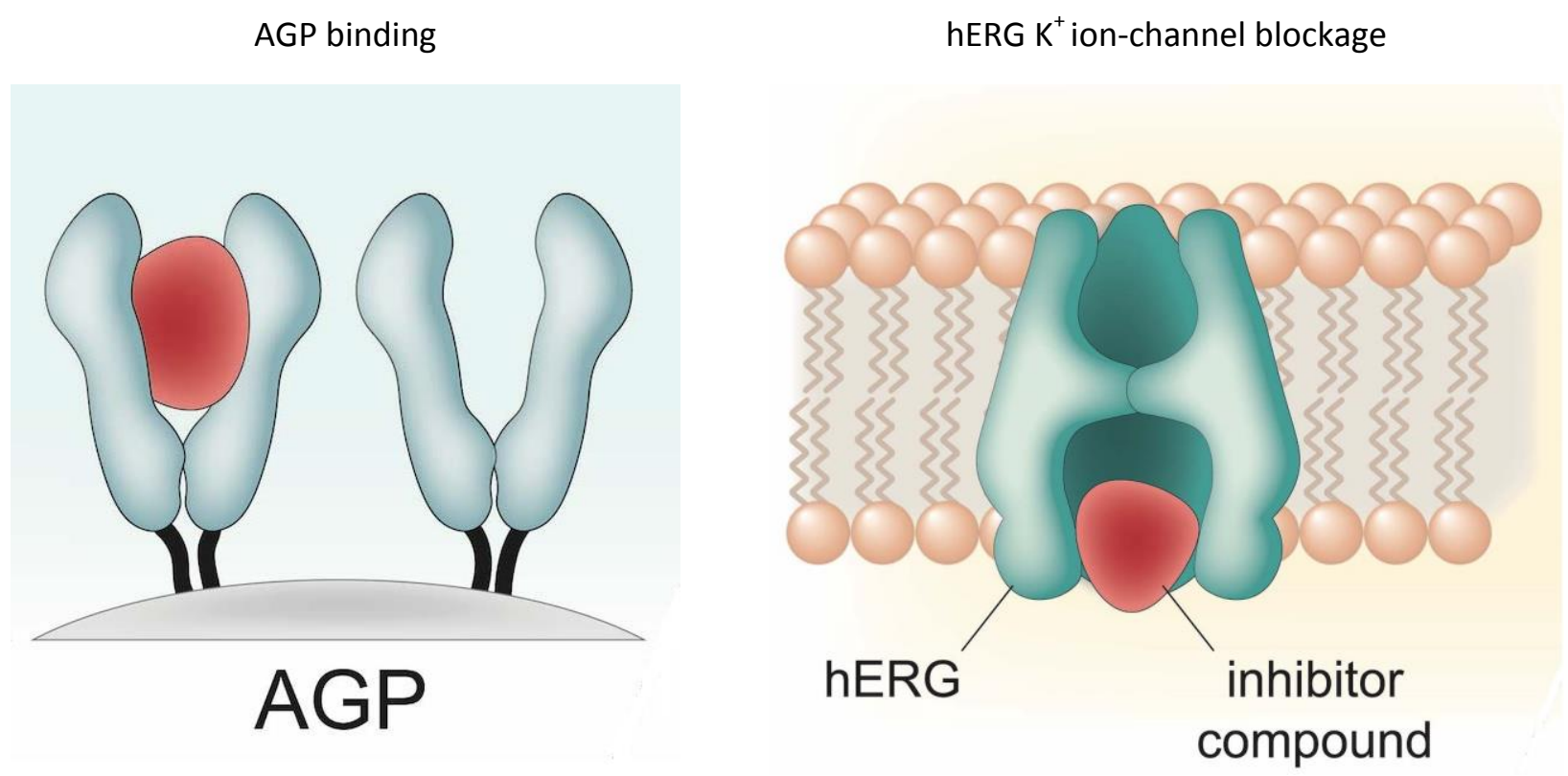

Figure 3. The similarity of the binding region of AGP and hERG potassium ion channels.

The steric structure and the negatively charged surface of AGP and the hERG ion channel suggest strong similarities. Compounds that block the channel have to penetrate the cell and have a relatively high concentration in the cell membrane where the potassium ion channel can be found [39]. This explains the importance of the membrane-binding properties in the model, as shown in Figure 4.

Validation of both models was performed by predictions of the 16 compounds included in the test set. The results are presented in Table 7. It can be seen that the prediction of the test set was superior in the case of the model derived with the measured properties, and the residuals did not exceed double the model error (0.693). On the other hand, predictions from the model derived with the calculated properties exhibited much worse residuals in most cases, with the $\mathrm{pIC}_{50}$ predictions of irbesartan and lamotrigine exceeding 1 log unit. 


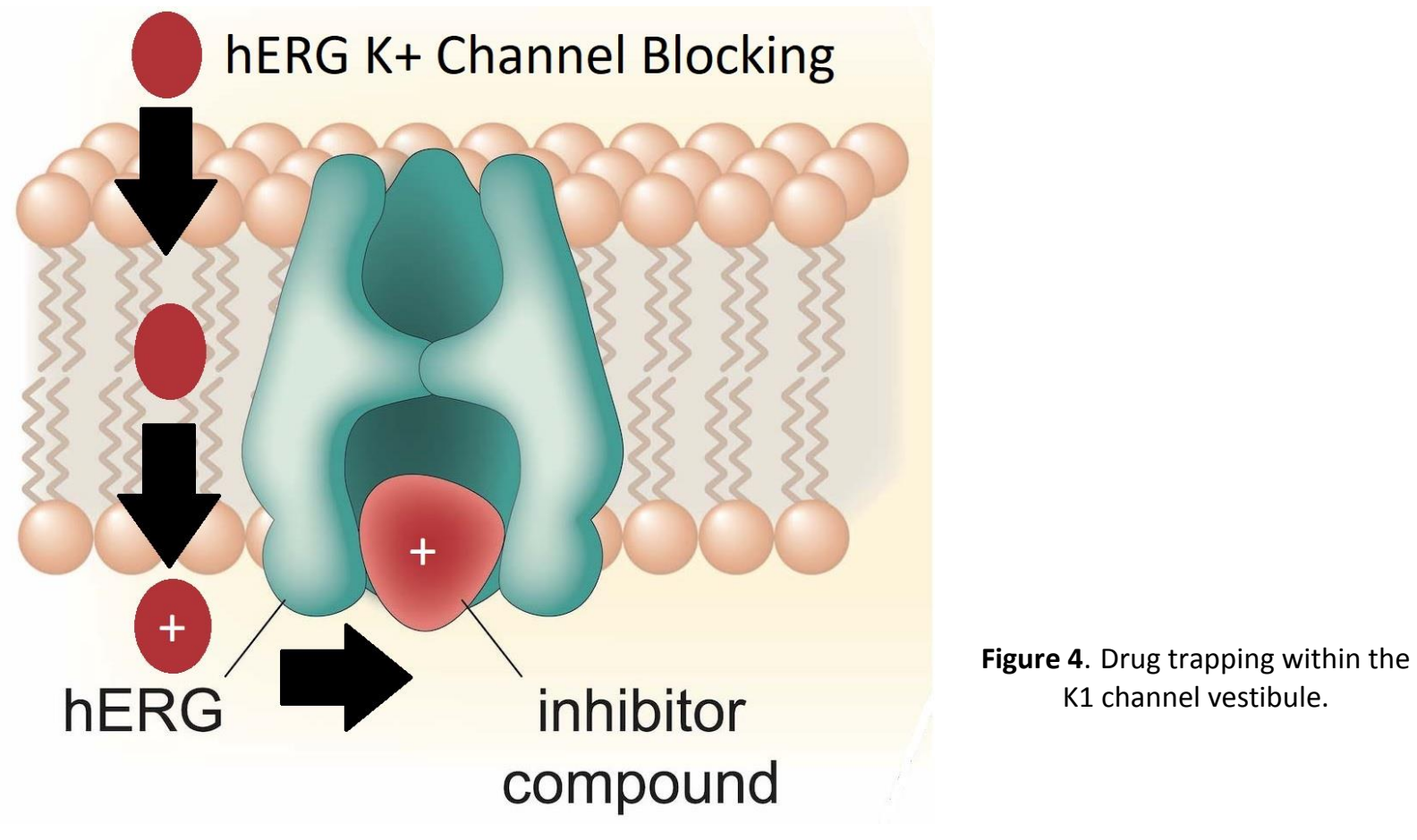

Table 7. The experimental and the predicted $\mathrm{pIC}_{50}$ values of the test set of compounds using the model described by equation 3 . The residuals show the difference between the measured and predicted values.

\begin{tabular}{|l|c|c|c|c|c|}
\hline \multirow{2}{*}{ Drug } & & \multicolumn{2}{c|}{$\begin{array}{c}\text { Model derived with } \\
\text { measured properties } \\
\text { (log } \boldsymbol{k} \text { AGP and log } \boldsymbol{k} \text { IAM) }\end{array}$} & \multicolumn{2}{c|}{$\begin{array}{c}\text { Model derived with } \\
\text { calculated properties } \\
\text { (log } \text { P and F-) }\end{array}$} \\
\cline { 2 - 6 } & $\begin{array}{c}\text { Experimental } \\
\text { pIC }_{50}\end{array}$ & $\begin{array}{c}\text { Predicted } \\
\text { pIC }\end{array}$ & Residual & $\begin{array}{c}\text { Predicted } \\
\text { pIC }_{50}\end{array}$ & Residual \\
\hline Alfuzosin & 4.28 & 4.74 & 0.46 & 3.85 & 0.42 \\
\hline Desloratadine & 5.75 & 5.78 & 0.04 & 5.51 & 0.24 \\
\hline Diphenhydramine & 4.94 & 5.02 & 0.08 & 5.23 & 0.29 \\
\hline Dofetilide & 5.72 & 4.96 & 0.76 & 4.85 & 0.87 \\
\hline Flecainide & 5.25 & 4.75 & 0.50 & 5.38 & 0.13 \\
\hline Fluoxetine & 5.73 & 5.74 & 0.01 & 5.65 & 0.08 \\
\hline Indomethacin & 3.72 & 4.55 & 0.83 & 4.08 & 0.36 \\
\hline Irbesartan & 4.71 & 5.28 & 0.57 & 5.73 & 1.02 \\
\hline Lamotrigine & 3.55 & 3.97 & 0.42 & 4.94 & 1.39 \\
\hline Metoprolol & 3.84 & 4.25 & 0.41 & 4.65 & 0.82 \\
\hline Protriptyline & 5.30 & 5.71 & 0.40 & 5.81 & 0.50 \\
\hline Sildenafil & 4.61 & 4.85 & 0.24 & 4.89 & 0.28 \\
\hline Spironolactone & 4.64 & 4.96 & 0.32 & 4.90 & 0.26 \\
\hline Trazodone & 5.30 & 4.84 & 0.46 & 5.38 & 0.08 \\
\hline Trimethoprim & 3.62 & 3.99 & 0.37 & 4.43 & 0.81 \\
\hline Ziprasidone & 6.44 & 5.69 & 0.75 & 5.69 & 0.75 \\
\hline
\end{tabular}




\section{Conclusions}

The hERG channel inhibition properties of drugs and drug discovery compounds are an important attribute as compounds with strong binding can cause cardiotoxic side effects during clinical trials. Early recognition of a compound's hERG inhibition potential is important to avoid the progression of compounds that fail later because of cardiotoxicity.

It has been demonstrated that two biomimetic HPLC property measurements can be used for screening molecules for hERG inhibition potential at an early stage of the drug discovery process. The model is based on the strong similarity between the AGP and hERG channel structures. Both attract positively charged compounds with a significant degree of lipophilicity. Both exhibit steric hindrance depending on the size and shape of the molecule being investigated. The membrane partition is also an important parameter as it reveals the membrane affinity of the compounds where the ion channel receptor is located. It has also been shown that two-dimensional physicochemical descriptors cannot provide an acceptable model for estimating the $\mathrm{hERG} \mathrm{pIC}_{50}$ of molecules.

Conflict of interest: Klara Valko is the founder of Biomimetic chromatography Ltd.

\section{References}

[1] I. Kola, J. Landis. Can the pharmaceutical industry reduce attrition rates?, Nat. Rev. Drug Discov. 3 (2004) 1-5. https://doi.org/10.1038/nrd1470.

[2] M.J. Waring, J. Arrowsmith, A.R. Leach, P.D. Leeson, S. Mandrell, R.M. Owen, G. Pairaudeau, W.D. Pennie, S.D. Pickett, J. Wang, O. Wallace, A. Weir. An analysis of the attrition of drug candidates from four major pharmaceutical companies. Nat. Rev. Drug Discov. 14 (2015) 475-486. https://doi.org/$10.1038 / \mathrm{nrd4609}$.

[3] F. Peter Guengerich. Mechanisms of drug toxicity and relevance to pharmaceutical development. Drug Metab. Pharmacokinet. 26 (2011) 3-14. https://doi.org/10.2133/dmpk.DMPK-10-RV-062.

[4] A. Casartelli, A. Lanzoni, R. Comelli, F. Crivellente, R. Defazio, R. Dorigatti, N. Fasdelli, I. Faustinelli, S. Pagliarusco, M. Tontodonati, P. Cristofori. A Novel and Integrated Approach for the Identification and Characterization of Drug-induced Cardiac Toxicity in the Dog. Toxicol. Pathol. 39 (2011) 361-371. https://doi.org/10.1177/0192623310390704.

[5] N. Ferri, P. Siegl, A. Corsini, J. Herrmann, A. Lerman, R. Benghozi. Drug attrition during pre-clinical and clinical development: Understanding and managing drug-induced cardiotoxicity. Pharmacol. Ther. 138 (2013) 470-484. https://doi.org/10.1016/i.pharmthera.2013.03.005.

[6] P.J. O'Brien. High-content analysis in toxicology: Screening substances for human toxicity potential, elucidating subcellular mechanisms and in vivo use as translational safety biomarkers. Basic Clin. Pharmacol. Toxicol. 115 (2014) 4-17. https://doi.org/10.1111/bcpt.12227.

[7] B.J. Ridder, D.J. Leishman, M. Bridgland-Taylor, et al. A systematic strategy for estimating hERG block potency and its implications in a new cardiac safety paradigm. Toxicol. Appl. Pharmacol. 394 (2020) 114961. https://doi.org/10.1016/i.taap.2020.114961.

[8] C. Kramer, B. Beck, J.M. Kriegl, T. Clark. A composite model for hERG blockade, ChemMedChem. 3 (2008) 254-265. https://doi.org/10.1002/cmdc.200700221.

[9] A.O. Aptula, M.T.D. Cronin. Prediction of hERG K+ blocking potency: Application of structural knowledge, SAR QSAR Environ. Res. 15 (2004) 399-411. https://doi.org/10.1080/10629360412331297353.

[10] E. Raschi, V. Vasina, E. Poluzzi, F. De Ponti. The hERG K+ channel: target and antitarget strategies in drug development. Pharmacol. Res. 57 (2008) 181-195. https://doi.org/10.1016/i.phrs.2008.01.009. 
[11] S. Munawar, M.J. Windley, E.G. Tse, M.H. Todd, A.P. Hill, J.I. Vandenberg, I. Jabeen. Experimentally validated pharmacoinformatics approach to predict hERG inhibition potential of new chemical entities. Front. Pharmacol. 9 (2018) 1-20. https://doi.org/10.3389/fphar.2018.01035.

[12] J.W. Warmke, B. Ganetzky. A family of potassium channel genes related to eag in Drosophila and mammals, Proc. Natl. Acad. Sci. U. S. A. 91 (1994) 3438-3442. https://doi.org/10.1073/pnas.91.8.3438.

[13] L. Du, M. Li, Q. You. The Interactions Between hERG Potassium Channel and Blockers. Curr. Top. Med. Chem. 9 (2012) 330-338. https://doi.org/10.2174/156802609788317829.

[14] J.T. Milnes, O. Crociani, A. Arcangeli, J.C. Hancox, H.J. Witchel. Blockade of HERG potassium currents by fluvoxamine: Incomplete attenuation by S6 mutations at F656 or Y652. Br. J. Pharmacol. 139 (2003) 887-898. https://doi.org/10.1038/sj.bjp.0705335.

[15] A.M. Aronov. Predictive in silico modeling for hERG channel blockers. Drug Discov. Today 10 (2005) 149-155. https://doi.org/10.1016/S1359-6446(04)03278-7.

[16] W.S. Redfern, L. Carlsson, A.S. Davis, W.G. Lynch, I. MacKenzie, S. Palethorpe, P.K.S. Siegl, I. Strang, A.T. Sullivan, R. Wallis, A.J. Camm, T.G. Hammond. Relationships between preclinical cardiac electrophysiology, clinical QT interval prolongation and torsade de pointes for a broad range of drugs: Evidence for a provisional safety margin in drug development. Cardiovasc. Res. 58 (2003) 3245. https://doi.org/10.1016/S0008-6363(02)00846-5.

[17] R. Netzer, A. Ebneth, U. Bischoff, O. Pongs. Screening lead compounds for QT interval prolongation. Drug Discov. Today 6 (2001) 78-84. https://doi.org/10.1016/S1359-6446(00)01602-0.

[18] C. Wood, C. Williams, G.J. Waldron. Patch clamping by numbers. Drug Discov. Today 9 (2004) 434441. https://doi.org/10.1016/S1359-6446(04)03064-8.

[19] R.J. Young, D.V.S. Green, C.N. Luscombe, A.P. Hill. Getting physical in drug discovery II : the impact of chromatographic hydrophobicity measurements and aromaticity. Drug Discov. Today 16 (2011) 822 830. https://doi.org/10.1016/i.drudis.2011.06.001.

[20] A. Fernández-Pumarega, B. Martín-Sanz, S. Amézqueta, E. Fuguet, M. Rosés. Estimation of the octanol-water distribution coefficient of basic compounds by a cationic microemulsion electrokinetic chromatography system. ADMET DMPK 8 (2020) 98-112. https://doi.org/10.5599/admet.760.

[21] C.A.S. Bergström, A. Avdeef. Perspectives in solubility measurement and interpretation. ADMET DMPK 7 (2019) 88-105. https://doi.org/10.5599/admet.686.

[22] K. Valko, S. Nunhuck, C. Bevan, M.H. Abraham, D.P. Reynolds. Fast Gradient HPLC Method to Determine Compounds Binding to Human Serum Albumin. Relationships with Octanol/Water and Immobilized Artificial Membrane Lipophilicity. J. Pharm. Sci. 92 (2003) 2236-48. https://doi.org/10.1002/ips.10494.

[23] K. Valko, C.M. Du, C.D. Bevan, D.P. Reynolds, M.H. Abraham. Rapid-gradient HPLC method for measuring drug interactions with immobilized artificial membrane: Comparison with other lipophilicity measures. J. Pharm. Sci. 89 (2000) 1085-96. https://doi.org/10.1002/15206017(200008)89:8<1085::aid-jps13>3.0.c0;2-n.

[24] S. Bunally, R.J. Young. The role and impact of high throughput biomimetic measurements in drug discovery. ADMET DMPK 6 (2018) 74-84. https://doi.org/10.5599/admet.530.

[25] G.H. Goetz, M. Shalaeva. Leveraging chromatography based physicochemical properties for efficient drug design. ADMET DMPK 6 (2018) 71-73. https://doi.org/10.5599/admet.529.

[26] F. Hollosy, K. Valko, A. Hersey, S. Nunhuck, G. Keri, C. Bevan. Estimation of Volume of Distribution in Humans from HPLC Measurements of Human Serum Albumin Binding and Immobilized Artificial Membrane Partitioning. J. Med. Chem. 49 (2006) 6958-6971. https://doi.org/10.1021/im050957i.

[27] K. Valko, E. Chiarparin, S. Nunhuck, D. Montanari. In vitro measurement of drug efficiency index to aid early lead optimization. J. Pharm. Sci. 101 (2012) 4155-69. https://doi.org/10.1002/ips.23305.

[28] S. Scheler, A. Fahr, X. Liu. Linear combination methods for prediction of drug skin permeation. ADMET DMPK 2 (2015) 199-220. https://doi.org/10.5599/admet.2.4.147. 
[29] C. Stephen, A. El Omri, L. Ciesla, Cellular membrane affinity chromatography (CMAC) in drug discovery from complex natural matrices. ADMET DMPK 6 (2018) 200-214. https://doi.org/10.5599/admet.535.

[30] P.C. Ray, M. Huggett, P.A. Turner, M. Taylor, L.A.T. Cleghorn et al. Spirocycle MmpL3 Inhibitors with Improved hERG and Cytotoxicity Profiles as Inhibitors of Mycobacterium tuberculosis Growth. ACS Omega 6 (2021) 2284-2311. https://doi.org/10.1021/acsomega.0c05589.

[31] F. Tsopelas, C. Stergiopoulos, A. Tsantili-Kakoulidou. Immobilized artificial membrane chromatography: From medicinal chemistry to environmental sciences. ADMET DMPK 6 (2018) 225241. https://doi.org/10.5599/admet.553.

[32] C.M. Du, K. Valko, C. Bevan, D. Reynolds, M.H. Abraham. Rapid Gradient RP-HPLC Method for Lipophilicity Determination: A Solvation Equation Based Comparison with Isocratic Methods. Anal. Chem. 70 (1998) 4228-4234. https://doi.org/10.1021/ac980435t.

[33] K. Valko, C.M. Du, C. Bevan, D.P. Reynolds, M.H. Abraham, Rapid method for the estimation of octanol/water partition coefficient (Log Poct) from gradient RP-HPLC retention and a hydrogen bond acidity term ( $(\alpha 2$ H). Curr. Med. Chem. 8 (2001) 1137-1146. https://doi.org/$10.2174 / 0929867013372643$.

[34] K.L. Valko, S.P. Teague, C. Pidgeon. In vitro membrane binding and protein binding (IAM MB/PB technology) to estimate in vivo distribution: applications in early drug discovery. ADMET DMPK 5 (2017) 14-38. https://doi.org/10.5599/admet.5.1.373.

[35] K. Valko. Handbook of Analytical Separations Volume 8, Separation Methods in Drug Synthesis and Purification. 2nd ed., Elsevier 2020 p.687. ISBN: 9780444640703.

[36] T. Sato, H. Yuki, K. Ogura, T. Honma. Construction of an integrated database for hERG blocking small molecules. PLoS One 13 (2018) 1-18. https://doi.org/10.1371/journal.pone.0199348.

[37] C. Stergiopoulos, D. Makarouni, A. Tsantili-Kakoulidou, M. Ochsenkühn-Petropoulou, F. Tsopelas. Immobilized artificial membrane chromatography as a tool for the prediction of ecotoxicity of pesticides. Chemosphere 224 (2019) 128-139. https://doi.org/10.1016/j.chemosphere.2019.02.075.

[38] R. Kaliszan, A. Nasal, M. Turowski. Quantitative structure-retention relationships in the examination of the topography of the binding site of antihistamine drugs on a1-acid glycoprotein. J. Chromatogr. A 722 (1996) 25-32. https://doi.org/10.1016/0021-9673(95)00523-4.

[39] M. Tristani-Firouzi, J. Chen, J.S. Mitcheson, M.C. Sanguinetti. Molecular biology of K+ channels and their role in cardiac arrhythmias. Am. J. Med. 110 (2001) 50-59. https://doi.org/10.1016/S00029343(00)00623-9. 